\title{
Molecular dynamics simulations of damage production by thermal spikes in Ge
}

\author{
Pedro López, ${ }^{\text {a) }}$ Lourdes Pelaz, Iván Santos, Luis A. Marqués, and María Aboy \\ Departamento de Electricidad y Electrónica, Universidad de Valladolid, E.T.S.I. Telecomunicación, \\ Valladolid 47011, Spain
}

(Received 28 November 2011; accepted 24 December 2011; published online 9 February 2012)

\begin{abstract}
Molecular dynamics simulation techniques are used to analyze damage production in Ge by the thermal spike process and to compare the results to those obtained for Si. As simulation results are sensitive to the choice of the inter-atomic potential, several potentials are compared in terms of material properties relevant for damage generation, and the most suitable potentials for this kind of analysis are identified. A simplified simulation scheme is used to characterize, in a controlled way, the damage generation through the local melting of regions in which energy is deposited. Our results show the outstanding role of thermal spikes in Ge, since the lower melting temperature and thermal conductivity of Ge make this process much more efficient in terms of damage generation than in Si. The study is extended to the modeling of full implant cascades, in which both collision events and thermal spikes coexist. Our simulations reveal the existence of bigger damaged or amorphous regions in $\mathrm{Ge}$ than in $\mathrm{Si}$, which may be formed by the melting and successive quenching induced by thermal spikes. In the particular case of heavy ion implantation, defect structures in Ge are not only bigger, but they also present a larger net content in vacancies than in $\mathrm{Si}$, which may act as precursors for the growth of voids and the subsequent formation of honeycomb-like structures. @ 2012 American Institute of Physics. [doi:10.1063/1.3682108]
\end{abstract}

\section{INTRODUCTION}

Germanium has attracted much attention in recent times, both in the microelectronic industry ${ }^{1,2}$ as a complementary material to $\mathrm{Si}$ and in radiation detection applications. ${ }^{3,4}$ This interest is motivated by its high carrier mobilities, ${ }^{5}$ the compatibility with $\mathrm{Si}$, which makes feasible the integration with Si-based technology, and its optimal properties for gammaray detection. ${ }^{3,4}$ The development of future high-mobility devices based on Ge requires basic research for process optimization, since the mechanisms of damage generation and amorphization in Ge associated to the introduction of dopants by ion implantation are not fully understood yet. High-purity $\mathrm{Ge}$ is commonly used in the fabrication of spectrometers for astrophysics and planetary space missions, as it allows a higher energy resolution at gamma-ray wavelengths than other detection media. ${ }^{3,4}$ The prolonged exposure to cosmic rays causes the degradation of Ge detectors, mainly due to the permanent displacement damage produced by $\mathrm{Ge}$ recoils resulting from the interactions with energetic protons. ${ }^{3} \mathrm{~A}$ better knowledge of the damage production mechanisms in $\mathrm{Ge}$ can contribute to predict $\mathrm{Ge}$ detectors degradation and to the development of "hardening" techniques against radiation.

Several experimental studies have been devoted to the analysis of damage generation and amorphization in $\mathrm{Ge}$, and in many of them, $\mathrm{Si}$ was used as a reference to compare with. Haynes et al. studied damage accumulation at different temperatures in $\mathrm{Si}_{1-\mathrm{x}} \mathrm{Ge}_{\mathrm{x}}$ layers in which the Ge fraction was varied. ${ }^{6}$ Increasing the Ge content resulted in a larger damage fraction, which was attributed to a less dynamic annealing in

a) Electronic mail: pedrol@ele.uva.es.
Ge caused by a reduction of defect mobility. A critical damage energy density of $5 \mathrm{eV} /$ atom for self-amorphization of Ge was calculated by Koffel et al., ${ }^{7}$ similar to the value found for Si. The higher nuclear energy deposition reported for $\mathrm{Ge}$ in comparison to $\mathrm{Si}$ was attributed to the higher mass and increased stopping power in Ge. ${ }^{8}$ Amorphization in Ge occurs at lower fluences than those required for $\mathrm{Si}^{6,9}$ and even a $5 \times 10^{13} \mathrm{~cm}^{-2}$ Ge self-implantation is able to amorphize. When heavy ions are implanted at high fluences, a severe swelling of amorphous Ge has been observed, the amorphous layer presenting a honeycomb-like structure formed by large cavities separated by thin walls. ${ }^{10-12}$ The formation and growth of voids inside damaged or amorphous regions is the main mechanism proposed to explain this effect. ${ }^{11,13}$ These unusual characteristics of amorphous layers in Ge have not been reported for $\mathrm{Si}$.

During ion implantation or irradiation processes, low energy interactions between the incident particle or recoil and lattice atoms result in local heating, which is referred to as the thermal spike phase. The local heating may even induce the melting of the region where energy is deposited. The reduced spatial and time scales of this process make very difficult its experimental observation. The molecular dynamics (MD) technique is a suitable tool to study the processes of damage generation, since it can simulate short lifetime events, and it allows identifying basic mechanisms and the extraction of parameters that can be used in continuum or atomistic simulators. ${ }^{14-17}$ As classical MD methods use semiempirical potentials to model the inter-atomic forces, the quality of simulation results is therefore linked to the accuracy of the potential to describe the material. The analysis of damage generation mechanisms in Ge and, in particular, 
the phenomenon of local melting requires that the chosen potential correctly describes not only the ballistic processes, but also the thermal properties of Ge.

Early studies of thermal spikes using MD simulations were performed for Si. De la Rubia et al. revealed that the solidification of the molten cascade core resulted in the formation of amorphous regions. ${ }^{16}$ Amorphous pockets may play an important role not only on damage accumulation and amorphization, but also on the performance of radiation detectors. A recent study points out that amorphous pockets are strong acceptors of electrons and may be responsible for the deleterious type inversion observed in Si detectors. ${ }^{18}$ Caturla et al. showed that the local melting process in Si presented a strong dependence on ion mass: it is almost negligible for light ions, but it may lead to the formation of large amorphous regions when heavy ions are implanted. ${ }^{17}$

Thermal spikes may be especially relevant in $\mathrm{Ge}$, due to its lower melting temperature. Nordlund et al. used MD simulations to analyze the damage production in a few collision cascades of recoils in $\mathrm{Ge}$ at $0 \mathrm{~K} .{ }^{14}$ At $\mathrm{keV}$ energies, they observed the existence of liquid-like regions that transformed into large damaged regions when the cascade cooled down. Almost all damage was located in large amorphous zones. A higher damage production yield was obtained in Ge than in $\mathrm{Si}$, which was explained by the effect of the lower melting point of Ge on damage generation by local melting. Nordlund et al. also showed that differences in the melting points obtained by several potentials resulted in variations on the amount of generated damage.

The development of atomistic models that can describe the local morphology of damage in Ge requires a detailed characterization of damage formation processes. In this study, classical MD simulations are used to characterize the damage generation during the thermal spike phase in Ge and to compare the results to those obtained for Si. The paper is organized as follows: In Sec. II, we estimate the relative weight that local melting has on damage production. The simulation scheme and the inter-atomic potentials most commonly used to describe Ge and Si are outlined in Sec. III. In Sec. IV, several potentials are evaluated in terms of material properties relevant for damage generation, and the most suitable ones are identified. In Sec. V, damage production by thermal spikes is analyzed by means of simplified MD simulations. The role of the local melting in real implant cascades as well as in the formation of big damage structures in Ge is studied in Sec. VI.

\section{ENERGY DISTRIBUTION IN DAMAGE GENERATION EVENTS}

During the implant process, the incoming ion transfers its energy to lattice atoms as it travels through the target, giving rise to the formation of defects and the heating of the lattice. A fraction of the ion energy is lost by inelastic stopping caused by the electron clouds surrounding the substrate atoms and by the background electron distribution in the material. When the ion collides with a target atom, a significant amount of energy is transferred to the nucleus. If the energy is high enough, the target atom may be permanently dis- placed from its lattice position. As a consequence, an interstitial and a vacancy are formed, which is known as a Frenkel pair. The minimum energy value required to create a Frenkel pair is referred to as the threshold displacement energy $\left(E_{d}\right)$. Sometimes the energy transferred in a collision is able to unbind an atom from its lattice position, but it is not enough to significantly separate the interstitial from the vacancy. For computational reasons, in many codes the just-created interstitial and the vacancy are automatically recombined. However, more detailed models consider that a metastable defect is formed, referred to as bond defect, which consists of a local rearrangement of bonds adding disorder to the lattice. ${ }^{19,20}$ The bond defect has been successfully used to model the formation and recrystallization of amorphous regions in $\mathrm{Si}^{21,22}$ Recent studies show that the bond defect is also formed in $\mathrm{Ge}$, presenting a similar stability to the bond defect in $\mathrm{Si}^{23}$ When the amount of energy transferred to a lattice atom is below $\mathrm{E}_{\mathrm{d}}$, no displacement or bond breaking takes place. Simulators based on the binary collision approximation (BCA) assume that this energy is lost to phonons and it is therefore neglected. However, the atoms which have received this residual energy, which we call hot particles (HPs), play also a role on damage generation, since the deposited energy increases the local temperature. As a result of the thermal spike and depending on the density of the deposited energy, the local temperature may overcome the melting point, resulting in the melting of the affected region. Subsequently, the heat quickly dissipates and the molten region cools down, giving rise to the formation of an amorphous region. ${ }^{16,24}$

The thermal spike process is closely related to the density of the deposited energy, which, from an atomistic point of view, is modeled by the number, energy, and distribution of HPs. The percentage of the total nuclear energy transferred to HPs can provide an estimation of the relative weight that local melting has on damage generation. For this purpose, we have analyzed the energy distribution in the different damage generation events by means of the simulator Ion Implantation in Semiconductors (IIS). ${ }^{25}$ This Monte Carlo code, based on the BCA, can simulate ion and recoils distributions, taking into account both elastic and inelastic stopping powers. $5 \mathrm{keV}$ cascades of $\mathrm{B}, \mathrm{Si}, \mathrm{Ge}$, and $\mathrm{Sb}$ ions were implanted in $\mathrm{Ge}$ and $\mathrm{Si}$ lattices. To have good statistics, 1000 cascades of each ion-substrate combination were considered. The energy loss in inelastic processes reaches approximately $50 \%$ for B implants, and it is progressively reduced for heavier ions to roughly $25 \%$ for Sb ions, presenting similar values in Ge and Si substrates. Table I reports the energy distribution only in elastic processes, which embrace the generation of Frenkel pairs (FPs), bond defects (BDs), and hot particles (HPs). HPs have been grouped following a criterion based on the identification of neighbors. The cut distance used to consider two particles as neighbors is the first minimum of the radial distribution function for the amorphous material at $300 \mathrm{~K}$. The average size and energy of the resulting HPs groups are also included in Table I.

Approximately, 25\% of the energy in elastic collisions results in the formation of Frenkel pairs, $20 \%$ is devoted to the creation of bond defects, and the remaining 55\% has 
TABLE I. Energy distribution percentages in damage generation events (Frenkel pairs (FPs), bond defects (BDs), and hot particles (HPs)) in elastic processes for $5 \mathrm{keV} \mathrm{B}, \mathrm{Si}, \mathrm{Ge}$, and $\mathrm{Sb}$ ions implanted in $\mathrm{Ge}$ and $\mathrm{Si}$ lattices. The average group size and energy for HPs groups is also shown.

\begin{tabular}{|c|c|c|c|c|c|c|c|c|c|c|}
\hline & \multicolumn{2}{|c|}{ FPs (\% energy) } & \multicolumn{2}{|c|}{ BDs (\% energy) } & \multicolumn{2}{|c|}{ HPs(\% energy) } & \multicolumn{2}{|c|}{ HPs group size } & \multicolumn{2}{|c|}{ HPs group energy $(\mathrm{eV})$} \\
\hline & $\mathrm{Ge}$ & $\mathrm{Si}$ & $\mathrm{Ge}$ & $\mathrm{Si}$ & $\mathrm{Ge}$ & $\mathrm{Si}$ & $\mathrm{Ge}$ & $\mathrm{Si}$ & $\mathrm{Ge}$ & $\mathrm{Si}$ \\
\hline B & 26 & 24 & 19 & 20 & 55 & 56 & 15 & 7 & 88 & 42 \\
\hline $\mathrm{Si}$ & 26 & 25 & 19 & 19 & 55 & 56 & 30 & 10 & 180 & 61 \\
\hline $\mathrm{Ge}$ & 26 & 24 & 20 & 20 & 54 & 56 & 54 & 19 & 334 & 116 \\
\hline $\mathrm{Sb}$ & 26 & 24 & 20 & 20 & 54 & 56 & 79 & 36 & 492 & 220 \\
\hline
\end{tabular}

been transferred to hot particles. Energy percentages are very similar for Ge and Si substrates, but HPs groups in Ge are notable bigger and with a higher energy than those formed in $\mathrm{Si}$. These results show that a significant fraction of energy is deposited on HPs and that there are differences between Ge and Si due to the distribution of HPs. The bigger size of HPs groups formed in Ge may make it easier to reach the high energy densities required to induce the local melting. It must also be noted that HPs group size increases with ion mass in both substrates, which may have implications in damage generation by heavy ion implantation.

The remarkable percentage of energy transferred to HPs underlines the importance that thermal spikes may have on damage generation. A detailed analysis of this process requires the use of MD simulations, since BCA codes do not consider energy transfers below $\mathrm{E}_{\mathrm{d}}$, which are fundamental to this mechanism.

\section{SIMULATION SCHEME}

The semiempirical potentials most commonly used in classical MD to describe Ge are the many-body potentials Tersoff and Stillinger-Weber. While there is only a parameter set for the Tersoff potential for $\mathrm{Ge},{ }^{26}$ several parameterizations of the Stillinger-Weber potential can be found in literature for this material. ${ }^{14,27,28}$ For $\mathrm{Si}$, a large number of potentials have been proposed. ${ }^{26,29-31}$ One of the main drawbacks of some of the semiempirical potentials available for $\mathrm{Ge}$ and $\mathrm{Si}$ is the high melting temperature obtained. Experimentally, melting occurs at $1211 \mathrm{~K}$ for $\mathrm{Ge}^{32}$ and at $1685 \mathrm{~K}$ for $\mathrm{Si}^{33}$ The Tersoff inter-atomic potential notably overestimates the melting temperature. Reported values in the literature obtained with this potential by different methods range from $2554 \mathrm{~K}$ to $3300 \mathrm{~K}$ for $\mathrm{Ge}^{34,35}$ and from $2000 \mathrm{~K}$ to $3000 \mathrm{~K}$ for $\mathrm{Si}^{14,19,34,36,37}$ The Stillinger-Weber potential, initially only proposed for $\mathrm{Si}$, was adapted by Ding et al. to model Ge, obtaining a very high melting temperature. ${ }^{27}$ Using the formulation of Ding et al. as a starting point, some authors have developed Stillinger-Weber-type potentials to obtain a more accurate description of the melting of $\mathrm{Ge}$, either focusing on reproducing the actual melting temperature $^{14}$ or the right cohesive energy. ${ }^{28}$ For $\mathrm{Si}$, the initial formulation of the Stillinger-Weber potential ${ }^{29}$ provides the $^{2}$ experimental melting temperature, $1700 \mathrm{~K}^{14}$

The potentials used in this study for Ge are multicomponent Tersoff $(\mathrm{T}-\mathrm{Ge})^{26}$ as well as three different parameterizations of Stillinger-Weber-type potentials: the initial parameter set proposed by Ding et al. (D-SW-Ge $)^{27}$ and the modifications introduced by Nordlund et al. (N-SW-Ge $)^{14}$ and by Posselt et al. (P-SW-Ge). ${ }^{28}$ For the modeling of Si, multi- component Tersoff $(\mathrm{T}-\mathrm{Si})^{26}$ and Stillinger-Weber $(\mathrm{SW}-\mathrm{Si})^{29}$ potentials are considered. Inter-atomic potentials are meant to describe the equilibrium properties of the materials they model. However, they do not accurately describe high-energy short-range interactions, which are important when simulating implant cascades. For this purpose, the Tersoff- and StillingerWeber-type potentials $\left(f_{\text {pot }}\right)$ are smoothly joined to the Ziegler-Biersack-Littmark (ZBL) repulsive inter-atomic potential $\left(\mathrm{f}_{\mathrm{ZBL}}\right)^{38}$ by means of a Fermi-like function $\mathrm{f}_{\mathrm{F}}\left(\mathrm{r}_{\mathrm{ij}}\right)$,

$$
\begin{gathered}
\text { Total potential }=f_{F}\left(r_{i j}\right) \cdot f_{\text {pot }}+\left(1+f_{F}\left(r_{i j}\right)\right) \cdot f_{Z B L}, \\
f_{F}\left(r_{i j}\right)=\frac{1}{1+e^{-A_{F}\left(r_{i j}-r_{C}\right)}},
\end{gathered}
$$

where $r_{i j}$ is the distance between the interacting particles, $A_{F}$ controls the abruptness of the transition between the two potentials, and $r_{C}$ indicates the transition point, i.e., the point at which each potential contributes as one-half of its value. For the description of Si by the Tersoff potential, the parameters used are the default values provided in the MD simulator Large-scale Atomic/Molecular Massively Parallel Simulator (LAMMPS). ${ }^{39}$ In the other cases, $A_{F}$ and $r_{C}$ have been calculated by ensuring that the transitions between the ZBL and the many-body potentials take place at a similar energy to that used for Tersoff Si. $\mathrm{r}_{\mathrm{C}}$ values were chosen so that the transition took place far enough from the attractive zone of the many-body potentials.

In Sec. IV, these potentials are evaluated in terms of relevant properties for damage generation. Classical molecular dynamics simulations are performed using the parallelrunning code LAMMPS. ${ }^{39}$

\section{DESCRIPTION OF FUNDAMENTAL PROPERTIES FOR DAMAGE GENERATION IN GE AND SI}

Local melting only takes place if the region in which energy is deposited remains above the melting temperature for enough time for the phase transformation to occur. ${ }^{24}$ Indeed, the process is a competition between the melting and the energy out-diffusion due to heat dissipation. The melting point and the thermal conductivity are the material parameters that most affect the thermal spike process and must be correctly described by the potential. The value of $E_{d}$ must also be evaluated, since this threshold energy determines the amount of primary radiation damage in the material, and it is a relevant parameter for BCA codes.

In Secs. IV A-C, the suitability of the potentials presented in Sec. III to model damage generation processes is 
checked and compared. The criteria used are the values of the displacement threshold energy, the melting temperature, and the thermal conductivity obtained with each potential.

\section{A. Displacement threshold energy}

Experimental and theoretical estimations of $\mathrm{E}_{\mathrm{d}}$ range from 15 to $30 \mathrm{eV}$ in $\mathrm{Ge}^{40}$ and from 10 to $30 \mathrm{eV}$ in $\mathrm{Si}^{41}$ In most BCA simulators, a value of $15 \mathrm{eV}$ is commonly assumed for both materials, providing a good description of damage. $^{25,38,42}$

In this work, the $\mathrm{E}_{\mathrm{d}}$ value for Ge and $\mathrm{Si}$ described by several potentials was calculated by means of MD simulations. Simulations were performed at $0 \mathrm{~K}$, in cubic cells containing 7840 atoms, in which periodic boundary conditions were applied in all directions. The cell size ensures that, for the maximum energy applied, the temperature remains low enough $(<70 \mathrm{~K})$ to discard any damage recovery. ${ }^{14} \mathrm{~A}$ kinetic energy ranging from 10 to $30 \mathrm{eV}$ was applied to one randomly chosen atom with velocity in a random direction. Simulations were run until the energy had spread and the cell had thermalized. Then, all atom positions were averaged for other 1000 time steps to remove atom vibrations. ${ }^{43}$ The final average positions were compared to those in the initial perfect lattice. Those atoms separated from a lattice site more than 0.125 times the lattice distance (approximately $0.71 \AA$ for $\mathrm{Ge}$ and $0.68 \AA$ for $\mathrm{Si}$ ) are identified as self-interstitials. Consequently, unoccupied lattice sites are considered vacancies. To improve statistics, the final number of self-interstitials was averaged over 200 simulations. Finally, $E_{d}$ is obtained as the value of the applied kinetic energy for which the average number of self-interstitials equals to unity.

Table II shows the values of $\mathrm{E}_{\mathrm{d}}$ obtained for $\mathrm{Ge}$ and $\mathrm{Si}$ described by the potentials mentioned before. As can be seen, kinetic energies in the range from 17 to $24 \mathrm{eV}$ are necessary to generate a Frenkel pair, which is in good agreement with the reported values in literature. For D-SW-Ge, the maximum kinetic energy applied $(30 \mathrm{eV})$ was not enough to generate on average one displaced atom, and, therefore, the $E_{d}$ value is beyond the energy range of this study.

\section{B. Melting temperature}

The different approaches and conditions used to determine the melting temperature by MD simulations has led to some discrepancy in the value of the melting point, even for the same inter-atomic potential. Our aim is to uniformize the calculation of the melting temperature by using the same method for the different potentials and substrates, so that a clear picture can be obtained on the differences on the melting point.

The simulation scheme to evaluate the melting temperature is based on the equilibrium between the crystalline and molten phases at the melting temperature at constant pressure and energy, as it has been described in detail elsewhere. ${ }^{19}$ Initially, the sample with the crystalline and molten regions is set to a temperature similar to the expected melting temperature. If the current temperature is above the melting point, additional melting takes place and the temperature is reduced. On the contrary, at a temperature below the melting point, the molten region at the interface recrystallizes, releasing heat, which increases the temperature. Thus, the system will evolve until the equilibrium between the two phases is reached, which occurs precisely at the melting temperature.

The calculated melting temperatures using this method are shown in Table II. For the Tersoff potential, melting temperatures are much higher than the experimental values both in $\mathrm{Ge}$ and $\mathrm{Si}$. In fact, the melting point for $\mathrm{Ge}$ is slightly higher than the one for Si, contrary to the experimental observations. This implies that the Tersoff potential may not correctly reproduce the differences between $\mathrm{Ge}$ and $\mathrm{Si}$ associated to thermal processes. As already mentioned, the Stillinger-Weber formulation for $\mathrm{Si}$ provides the accurate melting temperature, whereas, for $\mathrm{Ge}$, there are significant differences, depending on the parameter set used. The formulation by Nordlund et al. is the one that most accurately describes the melting point for Ge, as it has been specifically designed to fit this experimental property.

\section{Thermal conductivity}

For the calculation of the thermal conductivity, we have used the direct method as described by Schelling et al. ${ }^{44}$ It is based on non-equilibrium MD simulations to obtain the thermal conductivity ( $k$ ) from the Fourier law,

$$
J=-k \frac{\partial T}{\partial x} .
$$

Similarly to the experimental procedure, a constant heat flux (J) is imposed by heating a region of the simulation box and by removing the same amount of heat from another region. As a result, a linear temperature gradient $(\partial T / \partial x)$ is obtained. To minimize finite size effects, the calculation is performed on thin simulation cells with different lengths (L), and extrapolation is done to an infinite length. Thermal conductivity is calculated at $500 \mathrm{~K}$, which is a temperature high enough to neglect quantum effects. ${ }^{44}$

TABLE II. Experimental and simulated values of the displacement threshold energy $\left(\mathrm{E}_{\mathrm{d}}\right)$, melting temperature ( $\left.\mathrm{T}_{\mathrm{m}}\right)$, and thermal conductivity (k) for Ge and Si. Ge was described by means of the Tersoff potential (T-Ge) and the Ding (D-SW-Ge), Nordlund (N-SW-Ge), and Posselt (P-SW-Ge) parameterizations of the Stillinger-Weber potential. For Si, Tersoff (T-Si) and Stillinger-Weber (SW-Si) potentials were used.

\begin{tabular}{|c|c|c|c|c|c|c|c|c|}
\hline & \multicolumn{5}{|c|}{ Germanium } & \multicolumn{3}{|c|}{ Silicon } \\
\hline & Expt. & $\mathrm{T}-\mathrm{Ge}$ & N-SW-Ge & P-SW-Ge & D-SW-Ge & Expt. & $\mathrm{T}-\mathrm{Si}$ & SW-Si \\
\hline $\mathrm{E}_{\mathrm{d}}(\mathrm{eV})$ & $15-30$ & 21.4 & 17.6 & 19.9 & $>30$ & $10-30$ & 21.7 & 24.0 \\
\hline $\mathrm{T}_{\mathrm{m}}(\mathrm{K})$ & 1211 & 2448 & 1211 & 1305 & 2885 & 1685 & 2431 & 1653 \\
\hline $\mathrm{k}(\mathrm{W} / \mathrm{cmK})$ & 0.33 & 0.69 & 0.72 & 0.84 & 1.42 & 0.78 & 1.46 & 1.74 \\
\hline
\end{tabular}


Thermal conductivity values obtained in our calculations are included in Table II. All of them are roughly twice the experimental value for $500 \mathrm{~K}$ (approximately $0.33 \mathrm{~W} / \mathrm{cmK}$ for Ge and $0.78 \mathrm{~W} / \mathrm{cmK}$ for $\mathrm{Si}),{ }^{45}$ but for D-SW-Ge, which is four times higher. Similar deviations have been reported by other authors using this method for $\mathrm{Si}^{44,46}$ and can be attributed to the fact that, in the natural material, defect scattering significantly reduces thermal conductivity. However, useful information can be extracted from a qualitative comparison. For both substrates, the Tersoff potential provides a lower thermal conductivity than Stillinger-Weber-type potentials. From the description of Ge by the different parameterizations of Stillinger-Weber-type potentials, it can be observed that both thermal conductivity and melting temperature present their minimum values for the parameter set of Nordlund et al., they increase for the potential proposed by Posselt et al., and they are remarkable higher for the formulation by Ding et al. Note that a lower value of both properties favors local melting, since it is easier to reach the melting point and heat dissipation is slower.

The comparative study that has been performed provides the guidelines to choose the most appropriate potentials for the analysis of damage generation. Among the potentials considered, the Stillinger-Weber-type formulation by Nordlund et al. for Ge and the description of Si by StillingerWeber provide values of $E_{d}$, melting temperature, and thermal conductivity in good agreement with the experimental ones. This means that the actual differences regarding these parameters for both materials are correctly described, which is a key point if damage generation processes in Ge are to be compared to those in $\mathrm{Si}$. Therefore, N-SW-Ge and SW-Si will be the potentials used in the following study divided into two parts. First, we characterize the efficiency of thermal spikes on damage generation in Ge. Then, we simulate full implant cascades and analyze some characteristics that may be related to the local melting process.

\section{THERMAL SPIKES}

From an atomistic point of view, during the thermal spike, some atoms receive enough energy to induce the local melting of the region. The efficiency of the thermal spike to produce damage can be evaluated in a controlled way by applying a certain amount of energy to atoms organized in spheres, for simplicity, and by quantifying the number of self-interstitials formed once the simulation cell has thermalized, as described by Santos et al. ${ }^{24}$ The sphere size and the initial energy applied to each atom are varied, accounting for the large variety of conditions that may exist in actual implant cascades.

Kinetic energies densities of 5, 10, 15, and $20 \mathrm{eV} /$ atom, with corresponding velocities in random directions, were applied to atoms arranged in spheres of 5, 10, 20, 40,60, and 200 atoms. These sphere sizes embrace the mean sizes of HPs groups obtained by BCA simulations and reported in Table I. Each sphere was located at the center of a cubic simulation cell with periodic boundary conditions in all directions. The cell size was selected according to the total amount of deposited energy, ranging from 30976 to 313344 atoms, in order to keep the temperature low enough to prevent dynamic annealing. Initial temperature was set at $0 \mathrm{~K}$. The simulation cell was assumed to have thermalized when the average maximum kinetic energy of any atom was below $0.08 \mathrm{eV}$. Lower threshold values do not significantly modify the results, but they notably increase calculation time. Additionally, atom positions were time averaged during other 1000 time steps and compared with the initial perfect lattice. Self-interstitials were identified by following the same criterion used in the calculation of the displacement threshold energy. Results were averaged over 100 simulations.

Figure 1 shows the final number of self-interstitials as a function of the initial energy density applied to the atoms in the sphere for Ge and Si. For clarity, only spheres from 20 to 200 atoms are included. Within each sphere size, data are fitted to straight lines. The curved lines included in the figure represent the energy density for which the number of final self-interstitials equals the number of initial energetic atoms. Below these lines, some of the atoms that received energy still remain in their lattice positions and have not been displaced. Above the curved lines, the initial energetic atoms have been displaced as well as some of the surrounding atoms, which implies that the molten region is larger than the initial excited region.

For equivalent deposited energy, the number of selfinterstitials is always larger in Ge than in Si. The linear fits of the previous plot relate the final number of self-interstitials $\left(\mathrm{N}_{\mathrm{I}}\right)$ to the energy density $(\rho)$ for each number of initial energetic atoms $(\mathrm{N})$ by means of the following equation: ${ }^{47}$

$$
N_{I}=\propto(N) \rho-\beta(N),
$$

where $\alpha(\mathrm{N})$ and $\beta(\mathrm{N})$ depend on the initial sphere size $\mathrm{N}$. This expression can be reformulated by the introduction of two parameters, initially defined by Santos et al. for $\mathrm{Si}^{47} \mathrm{~A}$ threshold energy density $\left(\mathrm{E}_{\mathrm{T}}\right)$ can be defined as the minimum energy density required to generate self-interstitials $\left(\mathrm{N}_{\mathrm{I}}>0\right)$ and can be written as $\mathrm{E}_{\mathrm{T}}(\mathrm{N})=\beta(\mathrm{N}) / \alpha(\mathrm{N})$. An additional

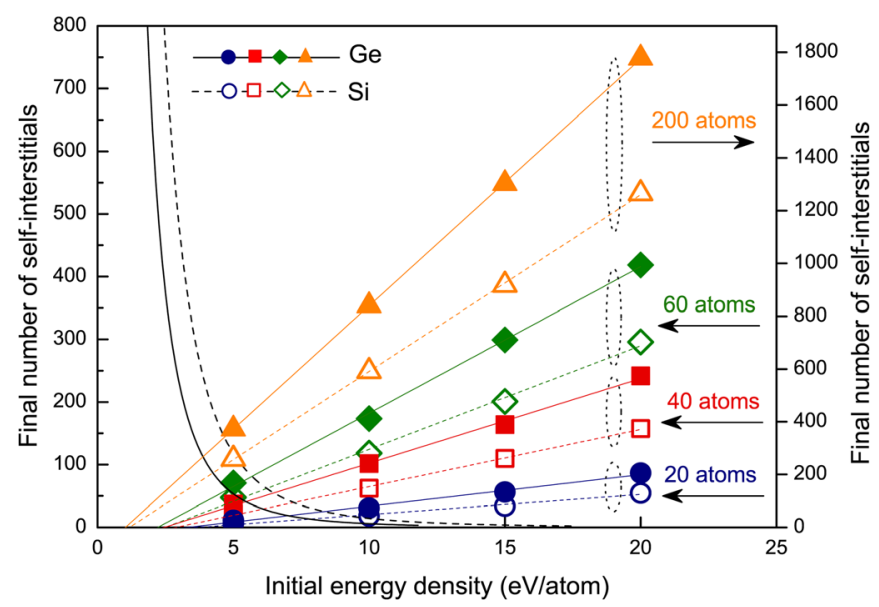

FIG. 1. (Color online) Number of self-interstitials as a function of the initial energy density for spheres with 20, 40, and 60 atoms (left axis) and 200 atoms (right axis). Solid symbols and lines represent Ge, while open symbols and dashed lines correspond to Si. Curved lines show the energy densities for which the number of self-interstitials equals the number of initial energetic atoms. 


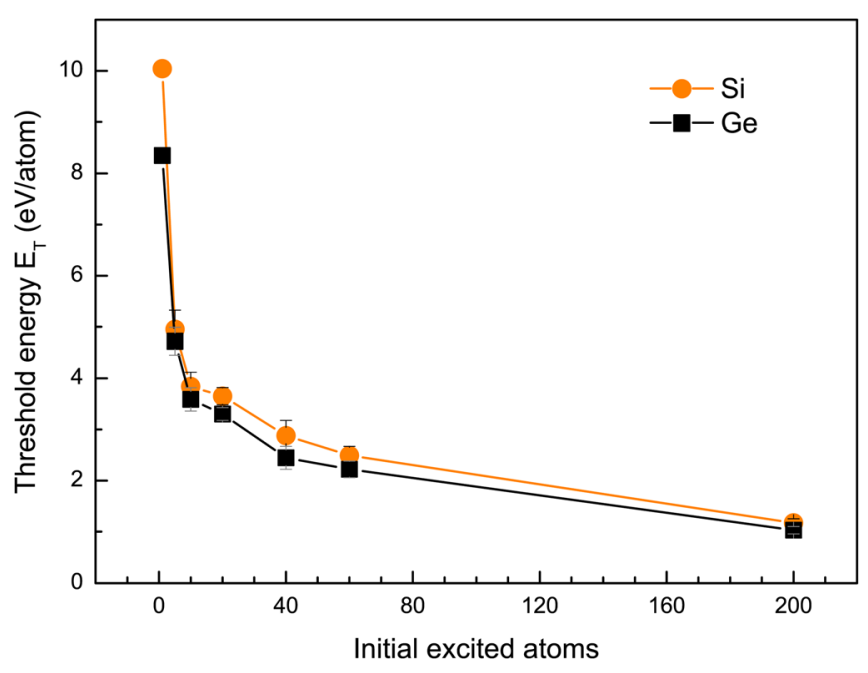

FIG. 2. (Color online) Threshold energy $\mathrm{E}_{\mathrm{T}}$ as a function of the number of initial excited atoms for $\mathrm{Ge}$ (squares) and $\mathrm{Si}$ (circles). The value for one excited atom has been extracted from the simulations of the threshold displacement energy. The lines are a guide to the eyes.

parameter, referred to as damage generation cost and defined as $\mathrm{D}_{\mathrm{C}}(\mathrm{N})=\mathrm{N} / \alpha(\mathrm{N})$, is a measure of the damage production yield: the lower the $\mathrm{D}_{\mathrm{C}}$, the easier damage generation is. For a particular value of $\mathrm{N}, \mathrm{D}_{\mathrm{C}}$ controls the number of selfinterstitials that will be generated by the energy exceeding $\mathrm{E}_{\mathrm{T}}$. Taking into account the definitions of $\mathrm{E}_{\mathrm{T}}$ and $\mathrm{D}_{\mathrm{C}}, \mathrm{Eq} .4$ can be rewritten as

$$
N_{I}=N \frac{\rho-E_{T}(N)}{D_{C}(N)} .
$$

Figures 2 and 3 report the values of $\mathrm{E}_{\mathrm{T}}$ and $\mathrm{D}_{\mathrm{C}}$ corresponding to the linear fits of the previous figure plus the data from the spheres of 5 and 10 atoms. For one atom, the values shown have been extracted from the simulations of the displacement threshold energy. Error bars correspond to the standard deviations calculated over 100 simulations. Although differences on the values of $E_{T}$ obtained for both materials are small, $\mathrm{D}_{\mathrm{C}}$

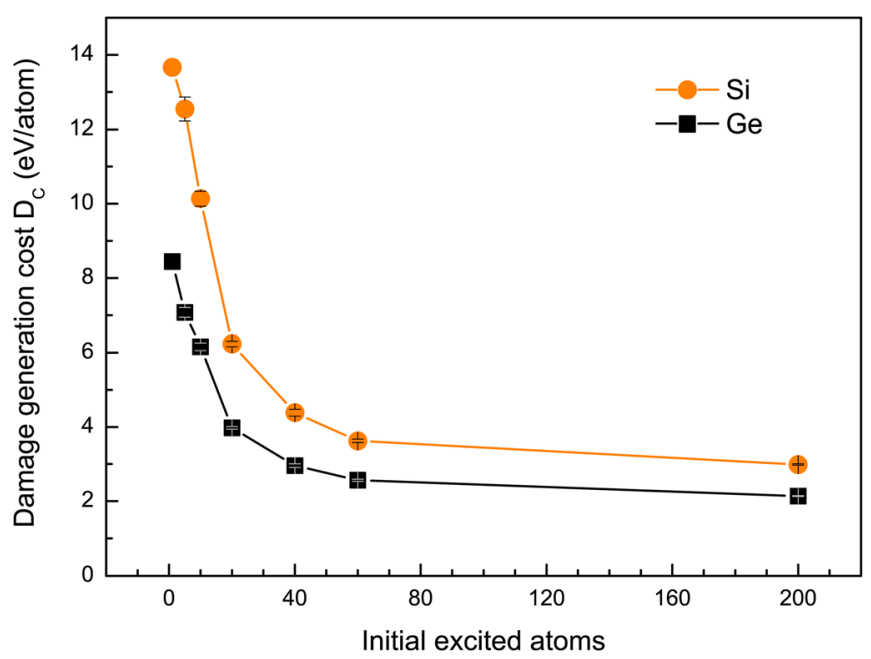

FIG. 3. (Color online) Damage generation cost $\mathrm{D}_{\mathrm{C}}$ as a function of the number of initial excited atoms for Ge (squares) and $\mathrm{Si}$ (circles). The value for one excited atom has been extracted from the simulations of the threshold displacement energy. The lines are a guide to the eyes. is significantly lower in $\mathrm{Ge}$ than in $\mathrm{Si}$. The figures clearly show that the values of $\mathrm{E}_{\mathrm{T}}$ and $\mathrm{D}_{\mathrm{C}}$ are not constant, but they are reduced as the number of initial energetic atoms increases. This behavior highlights the importance of the collective effect of the surrounding atoms, since it is easier to disorder an atom when its neighboring atoms are also excited.

The energy per atom required to generate as many selfinterstitials as initial excited atoms $\left(\mathrm{N}_{\mathrm{I}}=\mathrm{N}\right)$ equals the sum of $E_{T}$ and $D_{C}$. Following the trend of $E_{T}$ and $D_{C}$, this energy density is reduced as the number of initial excited atoms increases. Thus, damage generation is more efficient for big groups of initial excited atoms as the energy density necessary to disorder each atom is lower. For example, for Ge, around $10 \mathrm{eV} /$ atom are required to disorder all atoms in spheres with 10 atoms, while only $5 \mathrm{eV} /$ atom are necessary for spheres with 60 atoms. Note that BCA simulations reported in Sec. II, in which only ballistic processes were considered, revealed that the groups of HPs (or initial excited atoms) were significantly bigger in $\mathrm{Ge}$ than in $\mathrm{Si}$.

The data presented in this section clearly show the higher efficiency of the thermal spike phase in Ge in comparison to Si. For a similar number of initial excited atoms, more self-interstitials are generated in Ge. As these simulations were performed at $0 \mathrm{~K}$, dynamical anneal effects can be neglected. Thus, a lower dynamic annealing in Ge cannot explain the differences found in the thermal spike process between $\mathrm{Ge}$ and $\mathrm{Si}$. The thermal properties of $\mathrm{Ge}$, especially the lower melting temperature and thermal conductivity, are probably the main reasons of this behavior.

The simulation scheme followed in the previous analysis is just a systematic way to characterize the thermal spike process. The parameters $\mathrm{E}_{\mathrm{T}}$ and $\mathrm{D}_{\mathrm{C}}$ have a direct application in the development of improved BCA models, in which low energy interactions are also considered. ${ }^{47}$ In Sec. VI, we analyze real implant cascades, in which ballistic collisions and local melting processes coexist.

\section{IMPLANT CASCADES}

An implant cascade begins with the collision of the energetic ion with a lattice atom. Some of the ion energy is transferred to the atom, and both the ion and the recoil may produce subsequent collisions and sub-cascades. Previous MD studies only compared a few recoil cascades in Ge and $\mathrm{Si}^{14,48}$ We present here a MD study of damage generation in implant cascades in $\mathrm{Ge}$ and $\mathrm{Si}$ by implanting $\mathrm{B}, \mathrm{Si}, \mathrm{Ge}$, and $\mathrm{Sb}$ ions. This allows us to analyze damage production not only by self-implantation, but also by the introduction of ions with rather different masses and to study the effect on ion mass on the morphology and amount of generated damage.

$5 \mathrm{keV} \mathrm{B}, \mathrm{Si}, \mathrm{Ge}$, and $\mathrm{Sb}$ ions were implanted at random positions of the surface with random directions and a tilt angle of $7^{\circ}$. This energy value is within the range of interest of microelectronic and radiation detection applications. Even under high energy irradiation, defect clusters which may degrade detector performance, usually referred to as "terminal subclusters," are produced when the recoil loses the last 5-10 keV of energy. ${ }^{49}$ Periodic boundary conditions were applied only to $\mathrm{Y}$ and $\mathrm{Z}$, but not to the implant direction X. SRIM ${ }^{38}$ simulations were used 
to estimate the simulation cell size for each ion type and substrate material in order to avoid the self-overlapping of cascades. Simulations were done at $0 \mathrm{~K}$ to prevent dynamic annealing and to ensure that the results of the characterization of damage are not affected by other mechanisms related to thermal annealing. Besides, the low temperature analysis is important for the study of Ge-based gamma-ray spectrometers, since their working temperature is usually below $100 \mathrm{~K}$ to reduce leakage currents, which makes dynamic annealing almost negligible. ${ }^{4,50}$ The last two atomic layers in the $\mathrm{X}$ direction were kept fixed to avoid any artificial movement of the atoms within the simulation box. The preceding four layers were maintained at $0 \mathrm{~K}$ by means of velocity rescaling to model heat diffusion to the bulk. Implant cascades were considered to be finished when the maximum kinetic energy of any atom averaged for 10000 time steps was below $0.8 \mathrm{eV}$. The time step was automatically adjusted according to the maximum kinetic energy of atoms within a range from 0.01 to $1 \mathrm{fs}$.

Defect identification was done using the criterion described before, considering a radius of 0.125 times the lattice distance. Once each cascade is finished, atom positions were averaged for 1000 additional steps and then compared to the perfect lattice. The deformation of the lattice due to the momentum transfer from the ion was taken into account. Data were averaged over 200 simulations to improve statistics.

We first analyze some general aspects of damage generation in implant cascades as an evaluation of the validity of our simulations. The number of self-interstitials generated in $\mathrm{Ge}$ and $\mathrm{Si}$ as a function of ion mass is shown in Fig. 4. Error bars correspond to the standard deviations calculated over 200 simulations. Consistently with experimental observations and our previous results, similar implant cascades (same ion type and energy) result in more damage in Ge than in Si. As expected, the amount of damage increases with ion mass, mainly for low and medium ion masses. The enhanced damage production yield in Ge compared to $\mathrm{Si}$ is attributed to its higher mass and larger stopping powers, which results in higher damage energy densities. ${ }^{8}$ This means that, in Ge, energy is predominantly deposited in a few big groups of par-

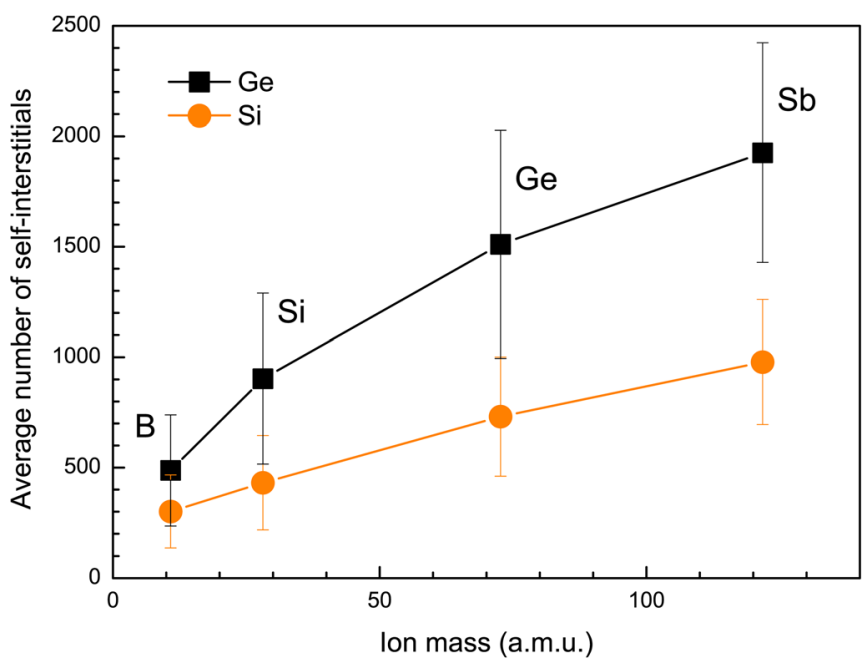

FIG. 4. (Color online) Average number of self-interstitials produced by implant cascades of $5 \mathrm{keV} \mathrm{B}, \mathrm{Si}, \mathrm{Ge}$, and $\mathrm{Sb}$ ions as a function of ion mass. $\mathrm{Ge}$ is represented by solid squares and $\mathrm{Si}$ by circles. ticles rather than in many small groups. This distribution, which is consistent with our BCA results, contributes to a more effective damage production process. Besides these differences between $\mathrm{Ge}$ and $\mathrm{Si}$, we have shown in this work that the thermal spike process, as one of the mechanisms responsible for damage production, is itself enhanced in Ge compared to $\mathrm{Si}$, and therefore, it may also contribute to the larger amount of damage reported for Ge.

Taking into account the total amount of energy deposited in the lattice and the number of self-interstitials generated, the average energy required to produce a self-interstitial can be estimated. As shown in Fig. 5, the average energy decreases as the ion mass increases, and it is always lower in Ge than in Si. These data allow us to analyze the role that ion mass plays on damage generation and, in particular, on thermal spike processes. As it can be seen, energy values associated to light ions, such as $B$, are close to the energy range of $E_{d}$, which means that damage generation is mainly governed by ballistic processes. When light ions are implanted, the energy is distributed in small regions along the cascade, as indicated by the size of HPs groups reported in Table I. In this scenario, most defects are produced by collisions, since the contribution of the thermal spike is small. On the contrary, for heavy ions, energy is deposited in bigger regions, which enhances the efficiency of the thermal spike. Local melting gives rise to a larger amount of damage than that produced by exclusively ballistic processes, which translates into an average energy per generated self-interstitial much lower than the value of $\mathrm{E}_{\mathrm{d}}$.

The thermal spike process is known to result in the formation of large damaged or amorphous regions both in $\mathrm{Ge}$ and in $\mathrm{Si}^{14,16,17}$ The distribution of the resulting damage after an implant cascade plays an important role on the survival and accumulation of damage and may provide insight into the role of the local melting on damage production. Theoretical and experimental observations in Si indicate that dilute damage is unstable and it easily annihilates due to dynamic annealing. ${ }^{17,51}$ On the contrary, compact and big damage structures are more likely to survive dynamic annealing and to accumulate, leading to the growth of bigger damaged

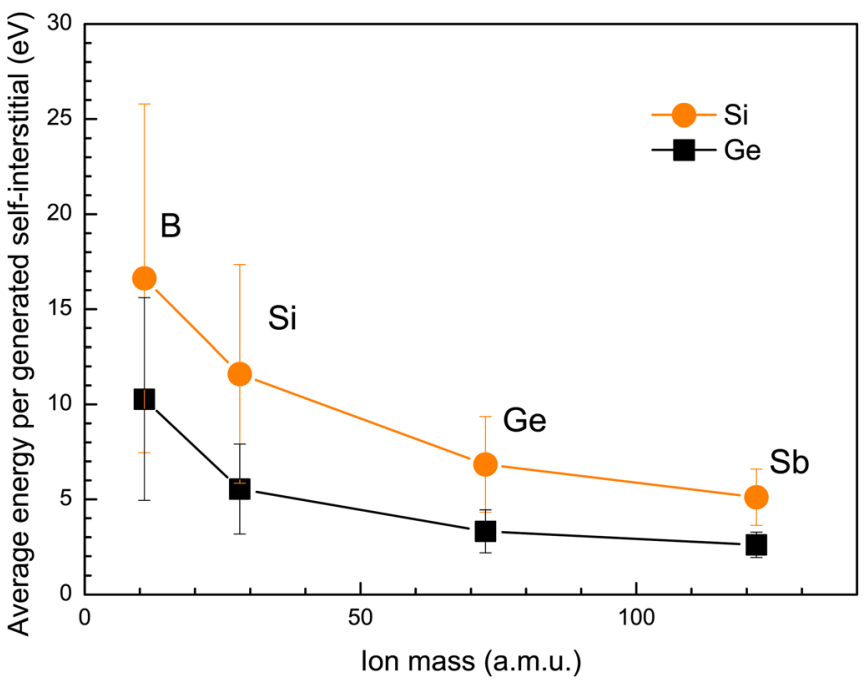

FIG. 5. (Color online) Average energy required to generate a self-interstitial in $\mathrm{Ge}$ and $\mathrm{Si}$ extracted from $5 \mathrm{keV} \mathrm{B}, \mathrm{Si}, \mathrm{Ge}$, and $\mathrm{Sb}$ implant cascades as a function of ion mass. Ge is represented by solid squares and Si by circles. 
regions or amorphous layers. ${ }^{17,51}$ To analyze the size of the damaged regions formed in our simulations, generated defects (both self-interstitials and vacancies) were grouped. We use a grouping criterion based on the identification of neighboring defects in three dimensions. The maximum distance at which two defects are considered as neighbors is the first minimum of the radial distribution function for the amorphous material at $300 \mathrm{~K}$. To belong to a group, a defect must be close to other defects already included in the group, and all its first neighbors must also be disordered. In this way, compact defect groups are formed, avoiding that a whole implant cascade is identified as a unique group.

Figure 6 reports the mean size of defect groups averaged over all groups formed in each of the 200 implant cascades. Implantation in Ge results in significantly bigger groups than those found in Si. The existence of bigger damage structures in $\mathrm{Ge}$ is related to the formation of molten regions in the cascade, ${ }^{14}$ which, once they have cooled down, remain as amorphous regions and may be a consequence of the outstanding role of the thermal spike process in this material. Damage produced in $\mathrm{Ge}$ is more localized than in $\mathrm{Si}$, which underlines the stronger tendency of Ge toward a direct impact amorphization ${ }^{14,15}$ by means of the overlapping of amorphous regions, especially under heavy ion implantation. In our simulations, performed at $0 \mathrm{~K}$, no dynamic annealing takes place, but, in a real scenario, a significant fraction of generated damage anneals out during the implant. Our results indicate that implant cascades in Ge do not only generate more damage than in $\mathrm{Si}$, but damage structures are bigger, which increases their stability and favor damage accumulation.

A particularly interesting phenomenon observed in Ge is the formation of honeycomb-like structures under ion irradiation. ${ }^{10,12}$ Significant swelling of the implanted region and the formation of cavities have been reported for fluences higher than $5 \times 10^{14}$ at $/ \mathrm{cm}^{2}$ when heavy ions are implanted. ${ }^{11,12}$ These structures appear inside amorphous regions and have been attributed to the formation and growth of voids. ${ }^{11,13}$ Some observations indicate that thermal activation is neces-

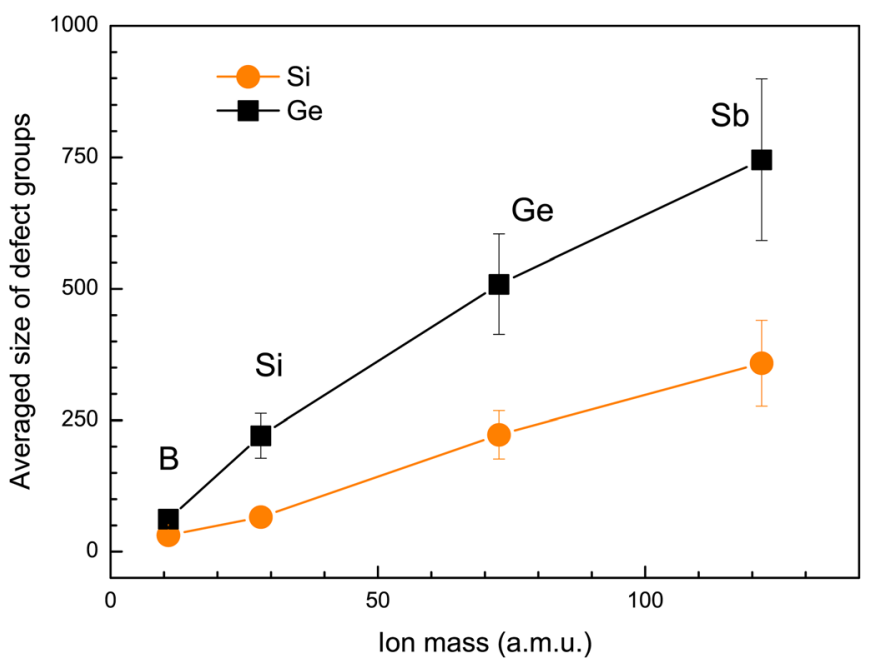

FIG. 6. (Color online) Average size of defect groups (average number of self-interstitials plus vacancies) formed in $5 \mathrm{keV} \mathrm{B}, \mathrm{Si}$, Ge, and $\mathrm{Sb}$ implant cascades as a function of ion mass. Ge is represented by solid squares and $\mathrm{Si}$ by circles.

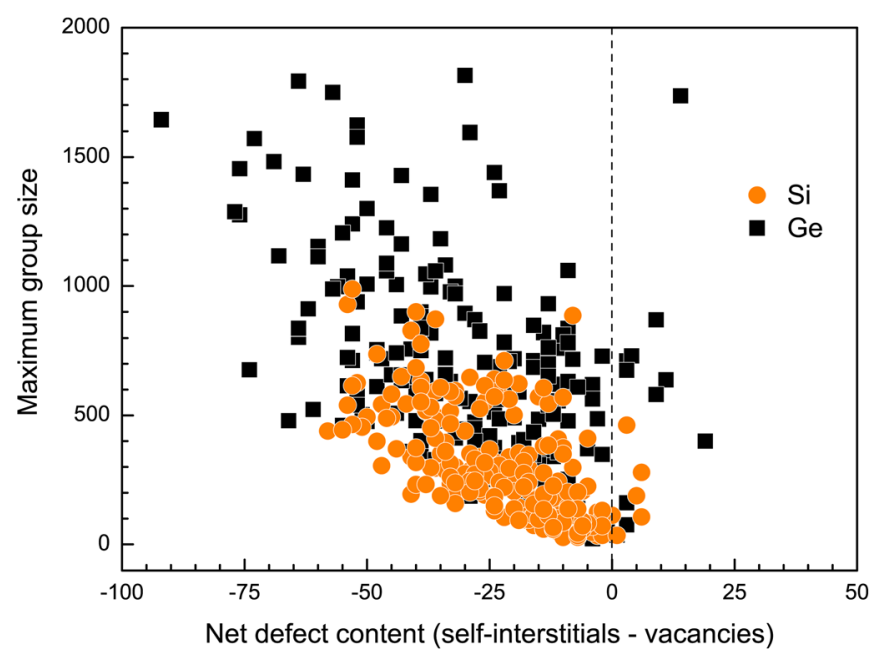

FIG. 7. (Color online) Maximum group size in each implant cascade of $\mathrm{Sb}$ ions in $\mathrm{Ge}$ and $\mathrm{Si}$ as a function of its net defect content (self-interstitials minus vacancies). Ge is represented by solid squares and Si by circles.

sary, as cavities only form for temperatures between $-50^{\circ} \mathrm{C}$ and $200^{\circ} \mathrm{C}^{10}$ This phenomenon is closely related to the existence of amorphous regions under heavy ion implantation in $\mathrm{Ge}$, and it has not been reported for $\mathrm{Si}$ in similar conditions. We have used our simulation results of $5 \mathrm{keV} \mathrm{Sb}$ implantation in Ge and Si to identify some characteristics of damage structures in Ge that could indicate the formation of cavities. Note that simulations were done at $0 \mathrm{~K}$, and therefore, the thermal component of this phenomenon is not considered. Defect grouping reveals that, for $\mathrm{Sb}$ implantation in both materials, a large damage structure is usually formed at the impact zone, composed by a high number of self-interstitials and vacancies. This amorphous structure is surrounded by small groups of defects. In each simulated cascade, the biggest group created has been identified and its net defect content calculated as the number of self-interstitials minus the number of vacancies. A positive result means that the group is rich in self-interstitials, whereas a negative value indicates an excess of vacancies. Figure 7 shows the size of the biggest group in each implant cascade as a function of its net defect content. As expected, they are notably big and, in most of them, vacancies are predominant. For Ge, groups have a larger number of defects and with a higher content on vacancies than those formed in $\mathrm{Si}$. These observations suggest that the presence in $\mathrm{Ge}$ of big damage structures rich in vacancies may act as precursors for the formation and growth of voids inside damaged regions.

\section{CONCLUSIONS}

Classical molecular dynamics techniques have been used to study and characterize damage generation by thermal spikes in Ge, analyzing the effects of this process on the amount and features of damage structures, and comparing the results to those obtained for $\mathrm{Si}$.

We have compared several inter-atomic potentials in terms of material properties relevant for damage formation, and we have identified the most suitable ones for the analysis of damage generation in $\mathrm{Ge}$ and $\mathrm{Si}$. The Stillinger-Weber description of $\mathrm{Si}$ and $\mathrm{Ge}$ (with the parameter set proposed by 
Nordlund et al.) provides right values of the displacement energy threshold, melting temperature, and thermal conductivity, which are key properties to correctly describe damage generation processes. Note that there is not a unique potential that accurately describes all the properties of $\mathrm{Ge}$ and $\mathrm{Si}$, and therefore, the study of other processes may require different potentials than the ones used in this work.

BCA simulations revealed that approximately 55\% of the total nuclear energy is deposited through low energy transfers, below the threshold energy necessary to produce a Frenkel pair, and it may contribute to local heating. Although the energy percentage is similar in $\mathrm{Ge}$ and $\mathrm{Si}$, the groups of particles that received energy below this threshold were significantly bigger in Ge.

The thermal spike process has been analyzed and characterized by means of simple simulation schemes and the definition of two parameters, $\mathrm{E}_{\mathrm{T}}$ and $\mathrm{D}_{\mathrm{C}}$, which are important for the development of improved BCA models. Our results evidence the relevance of this mechanism in damage production in $\mathrm{Ge}$ by the melting and subsequent amorphization of regions in which energy is deposited. The lower melting point and thermal conductivity of $\mathrm{Ge}$ make this process much more efficient than in Si. Besides, the existence of bigger groups of initial excited atoms in $\mathrm{Ge}$ increases the yield of this process, since it reduces the energy per atom required to generate damage.

The simulation of implant cascades clearly shows a larger amount of damage and bigger defect structures in Ge than in $\mathrm{Si}$. These extensive damaged regions may be produced by the local melting of the cascade core as a consequence of the outstanding role of the thermal spike process in Ge. Heavy ion implantation in Ge results in large defect groups with a higher net content in vacancies than those formed in $\mathrm{Si}$, which may act as precursors for the growth of voids inside damaged regions and the subsequent formation of honeycomb-like structures.

\section{ACKNOWLEDGMENTS}

This work has been funded by the Spanish DGI under project TEC2008-06069 and the JCyL Consejería de Educación y Cultura under project VA011A09. The authors want to thank Dr. J. M. Hernández-Mangas for his help on the use of the simulation code IIS.

${ }^{1}$ C. Claeys and E. Simoen, Germanium-Based Technologies - From Materials to Devices (Elsevier, Amsterdam, 2007).

${ }^{2}$ M. Caymax, G. Eneman, F. Bellenger, C. Merckling, A. Delabie, G. Wang, R. Loo, E. Simoen, J. Mitard, B. de Jaeger, G. Hellings, K. de Meyer, M. Meuris, and M. Heyns Tech. Dig. - Int. Electron Devices Meet. 461 (2009).

${ }^{3}$ A. Owens, S. Brandenburg, E.-J. Buis, H. Kiewiet, S. Kraft, R. W. Ostendorf, A. Peacock, F. Quarati, and P. Quirin, Nucl. Instrum. Methods Phys. Res. A 583, 285 (2007).

${ }^{4}$ P. Kurczynski, R. H. Pehl, E. L. Hull, D. Palmer, M. J. Harris, H. Seifert, B. J. Teegarden, N. Gehrels, T. L. Cline, R. Ramaty, D. Sheppard, N. W. Madden, P. N. Luke, C. P. Cork, D. A. Landis, D. F. Malone, and K. Hurley, Nucl. Instrum. Methods Phys. Res. A 431, 141 (1997).

${ }^{5}$ S. M. Sze and J. C. Irvin, Solid State Electron 11, 599 (1968).

${ }^{6}$ T. E. Haynes and O. W. Holland, Appl. Phys. Lett. 61, 61 (1992).

${ }^{7}$ S. Koffel, P. Scheiblin, A. Claverie, and G. BenAssayag, J. Appl. Phys. 105, 013528 (2009).

${ }^{8}$ E. Rimini, Ion Implantation: Basics to Device Fabrication (Kluwer Academic, Boston, 1995).
${ }^{9}$ D. Y. C. Lie, A. Vantomme, F. Eisen, T. Vreeland, Jr., M.-A. Nicolet, T. K. Carns, V. Arbet-Engels, and K. L. Wang, J. Appl. Phys. 74, 6039 (1993).

${ }^{10}$ B. Strizker, R. G. Elliman, and J. Zou, Nucl. Instrum. Methods Phys. Res. B 175, 193 (2001).

${ }^{11}$ T. Janssens, C. Huyghegebaert, D. Vanhaeren, G. Winderickx, A. Satta, M. Meuris, and W. Vandervorst, J. Vac. Sci. Technol. B 24, 510 (2006).

${ }^{12}$ R. J. Kaiser, S. Koffel, P. Pichler, A. J. Bauer, B. Amon, A. Claverie, G. BenAssayag, P. Scheiblin, L. Frey, and H. Ryssel, Thin Solid Films 518, 2325 (2010).

${ }^{13}$ N. Nitta, M. Taniwaki, Y. Hayashi, and T. Yoshije, J. Appl. Phys. 92, 1799 (2002).

${ }^{14}$ K. Nordlund, M. Ghaly, R. S. Averback, M.-J. Caturla, T. Diaz de la Rubia, and J. Tarus, Phys. Rev. B 57, 7556 (1998).

${ }^{15}$ J. Nord, K. Nordlund, and J. Keinonen, Phys. Rev. B 65, 165329 (2002).

${ }^{16}$ T. Diaz de la Rubia and G. H. Gilmer, Phys. Rev. Lett. 74, 2507 (1995).

${ }^{17}$ M.-J. Caturla, T. Diaz de la Rubia, L. A. Marqués, and G. H. Gilmer, Phys. Rev. B 54, 16683 (1996).

${ }^{18}$ E. Holmström, K. Nordlund, and M. Hakala, Phys. Rev. B 82, 104111 (2010).

${ }^{19}$ L. A. Marqués, L. Pelaz, J. Hernández, J. Barbolla, and G. H. Gilmer, Phys. Rev. B 64, 045214 (2001).

${ }^{20}$ M. Tang, L. Colombo, J. Zhu, and T. Diaz de la Rubia, Phys. Rev. B 55, 14279 (1997).

${ }^{21}$ L. Pelaz, L. A. Marqués, M. Aboy, J. Barbolla, and G. H. Gilmer, Appl. Phys. Lett. 82, 2038 (2003).

${ }^{22}$ P. Lopez, L. Pelaz, L. A. Marqués, and I. Santos, J. Appl. Phys. 101, 093518 (2007).

${ }^{23}$ P. López, L. Pelaz, L. A. Marqués, I. Santos, and M. Aboy, private communication (2011).

${ }^{24}$ I. Santos, L. A. Marqués, and L. Pelaz, Phys. Rev. B 74, 174115 (2006).

${ }^{25}$ J. M. Hernández-Mangas, J. Arias, L. Bailón, M. Jaraiz, and J. Barbolla, J. Appl. Phys. 91, 658 (2002).

${ }^{26}$ J. Tersoff, Phys. Rev. B 39, 5566 (1989).

${ }^{27}$ K. Ding and H. C. Andersen, Phys. Rev. B 34, 6987 (1986).

${ }^{28}$ M. Posselt and A. Gabriel, Phys. Rev. B 80, 045202 (2009).

${ }^{29}$ F. H. Stillinger and T. A. Weber, Phys. Rev. B 31, 5262 (1985).

${ }^{30}$ J. F. Justo, M. Z. Bazant, E. Kaxiras, V. V. Bulatov, and S. Yip, Mater. Res. Soc. Symp. Proc. 469, 217 (1997).

${ }^{31}$ T. J. Lenosky, B. Sadigh, E. Alonso, V. V. Bulatov, T. Diaz de la Rubia, J. Kim, A. F. Voter, and J. D. Kress, Modell. Simul. Mater. Sci. Eng. 8, 825 (2000).

${ }^{32}$ CRC Handbook of Chemistry and Physics, 87 th ed., edited by D. R. Lide (CRC, Boca Raton, 2006).

${ }^{33}$ J. W. Mayer and S. S. Lau Electronics Materials Science for Integrated Circuits in Si and GaAs (MacMillan, New York, 990).

${ }^{34}$ S. J. Cook and P. Clancy, Phys. Rev. B 47, 7686 (1993).

${ }^{35}$ J. K. Bording, Phys. Rev. B 62, 7103 (2000).

${ }^{36}$ K. Nordlund and R. S. Averback, Phys. Rev. B 56, 2421 (1997).

${ }^{37}$ J. Tersoff, Phys. Rev. B 38, 9902 (1988).

${ }^{38}$ J. F. Ziegler, J. P. Biersack, and U. Littmark The Stopping and Range of Ions in Solids, Stopping and Ranges on Ions in Matter (Pergamon, New York, 1984), Vol. 1.

${ }^{39}$ See http://lammps.sandia.gov / for information on LAMMPS simulator.

${ }^{40}$ E. Holmström, K. Nordlund, and A. Kuronen, Phys. Scr. 81, 035601 (2010).

${ }^{41}$ E. Holmström, A. Kuronen, and K. Nordlund, Phys. Rev. B 78, 045202 (2008).

${ }^{42}$ M. T. Robinson and I. M. Torrens, Phys. Rev. B 9, 5008 (1974).

${ }^{43}$ L. A. Marqués, L. Pelaz, P. Castrillo, and J. Barbolla, Phys. Rev. B 71, 085204 (2005).

${ }^{44}$ P. K. Schelling, S. R. Phillpot, and P. Keblinski, Phys. Rev. B 65, 144306 (2002).

${ }^{45}$ C. J. Glassbrenner and G. A. Slack, Phys. Rev. 134, 1058 (1964).

${ }^{46}$ Y. Lee, S. Lee, and G. S. Hwang, Phys. Rev. B 83, 125202 (2011).

${ }^{47}$ I. Santos, L. A. Marqués, L. Pelaz, and P. Lopez, J. Appl. Phys. 105, 083530 (2009).

${ }^{48}$ J. Nord, K. Nordlund, and J. Keinonen, Nucl. Instrum. Methods Phys. Res. B 193, 165329 (2002).

${ }^{49}$ J. R. Srour, C. J. Marshall, and P. W. Marshall, IEEE Trans. Nucl. Sci. 50, 653 (2003).

${ }^{50}$ H. G. Thomas, J. Eberth, F. Becker. T. Burkardt, S. Freund, U. Hermkens, T. Mylaeus, S. Skoda, W. Teichert, A. v.d Werth, P. von Brentano, M. Berst, D. Gutknecht, and R. Henck, Nucl. Instrum. Methods Phys. Res. A 332, 215 (1993).

${ }^{51}$ E. C. Baranova, V. M. Gusev, Yu. V. Martynenko, C. V. Starinin, and I. B. Haibullin, Radiat. Eff. 18, 21 (1973). 